\title{
Design of Eddy Current Testing Probe for Surface Defect Evaluation
}

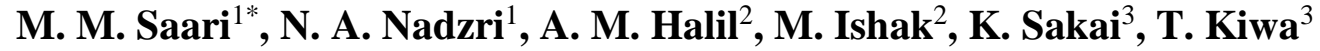 \\ and K. Tsukada ${ }^{3}$ \\ ${ }^{1}$ Faculty of Electrical \& Electronics Engineering, Universiti Malaysia Pahang, \\ 26600 Pekan, Pahang, Malaysia \\ *Email: mmawardi@ump.edu.my \\ Phone: +6094246119; Fax: +6094246111 \\ ${ }^{2}$ Faculty of Mechanical Engineering, University Malaysia Pahang, \\ Pekan Campus, 26600 Pekan, Pahang, Malaysia \\ ${ }^{3}$ Graduate School of Interdisciplinary Science and Engineering in Health Systems, \\ Okayama University, Okayama 700-8530, Japan
}

\begin{abstract}
Early detection of defects in metallic components used in infrastructure is crucial to ensure their safety and reliability. This paper presents a development of a small eddy current testing (ECT) probe for evaluation of sub-millimetre surface defects. The ECT probe is developed in a planar differential using sensitive anisotropy magnetoresistance sensors, and the signal amplification is achieved by a home-made instrumentation amplifier. The developed ECT probe is evaluated by performing phase sensitive measurement of the magnetic responses of sub-millimetre surface slits at the excitation field of $200 \mathrm{~Hz}$ and $10 \mathrm{kHz}$. Compared to the real component of the magnetic response, the imaginary component can be used to identify the existence and position of the slits based on the signal intensity change caused by the induced eddy current. The spatial distribution of the magnetic response measured by the ECT probe can be used to estimate the dimension of the slit. It is expected that the developed ECT probe can be utilised for assessment of sub-millimetre surface defect.
\end{abstract}

Keywords: Eddy current testing (ECT); crack detection; gradiometer; ferromagnetic material; Anisotropic Magnetoresistance (AMR) sensor.

\section{INTRODUCTION}

Nondestructive testing (NDT) serves as a crucial process to ensure safety and reliability in metallic materials that are being used in important applications such as infrastructure. Crack in metallic parts is one of the defects that can lead to fatal accidents where early detection of it is crucial to prevent accidents. Cracking can arise in a uniform metallic material due to the fatigue caused by the exposure of continues stress and strain on it [1, $2]$, and in weld areas where metallic parts are joined together by the welding processes $[3,4]$. Furthermore, it is reported that welding defects are the main cause of the catastrophic failure of the metallic structures such as in the gas pipeline system [5] and obtaining the optimised parameters for each welding process is one of the crucial steps to prevent defects [6-8]. It is worth to note that, cracking in the welding area can occur during and after the welding process due to hot and cold cracking, the formation of cavities, impurities inclusions such as oxides and non-metallic slag, lack of fusion, 
incomplete penetration and undercut [4]. Moreover, arise of fatigue cracks is also possible in a welded component when it is exposed to fatigue loads.

To detect defects such as crack, a variety of NDT techniques have been developed and used in industries for inspection of the surface, internal and backside defects such as magnetic testing, visual inspection, radiographic (X-ray) testing, and ultrasonic testing $[2,5,9]$. Each method has its advantages such as the visual inspection is simple, quick and relatively inexpensive; however, this method is limited to surface defects and requires experienced inspectors [2]. In the case of X-ray and ultrasonic testing, sub-surface defects can be assessed owing to the penetration of high energy electromagnetic and sound waves, respectively. Compared to the X-ray method, ultrasonic testing is not harmful, and the testing equipment is more compact, making it easier to be applied at the field test. However, ultrasonic testing requires trained operators for a precise assessment of defects [10]. Since metallic materials are conductive and possess strong magnetic properties, the defect inspection using the magnetic method is one of the promising techniques owing to its safe, fast and non-contact nature. Furthermore, its measurement system is also simple and can be compacted easily [11]. The common measurement technique using the magnetic method includes measurement of induced eddy current in the metallic parts by AC magnetic field, namely, eddy current testing (ECT) $[2,12,13]$, pulse eddy current (PEC) testing [14-16] and measurement of magnetic flux leakage from the metallic parts during application of external magnetic fields, namely, magnetic flux leakage (MFL) testing $[1,17]$.

In a typical ECT system, an excitation coil is used to induced eddy current while magnetic sensors such as detection coils, Hall [18], Tunnelling Magnetoresistance (TMR) [9, 19] and Anisotropy Magnetoresistance (AMR) sensors [20, 21] and Superconducting Quantum Interference Devices (SQUID) [22, 23] are utilised. In this method, the utilisation of the electromagnetic wave penetration depth governed by the so-called skin effect enables the detection of the buried defects. When an excitation magnetic field $B_{0} e^{j 2 \pi f t}$ is applied perpendicularly to a conductive material that lies in the $x y$ plane, based on Maxwell's equations, the induced eddy current components are given by:

$$
\begin{aligned}
& \mathrm{J}_{\mathrm{x}}=\mathrm{J}_{\mathrm{x} 0} \mathrm{e}^{-(\pi \mathrm{f} \mu \sigma)^{\frac{1}{2}} \mathrm{z}} \mathrm{e}^{\mathrm{j}\left(2 \pi \mathrm{ft}+\alpha_{0}-(\pi \mathrm{f} \mu \sigma)^{\frac{1}{2}} \mathrm{z}\right)} \\
& \mathrm{J}_{\mathrm{y}}=\mathrm{J}_{\mathrm{y} 0} \mathrm{e}^{-(\pi \mathrm{f} \mu \sigma)^{\frac{1}{2}} \mathrm{z}} \mathrm{e}^{\mathrm{j}\left(2 \pi \mathrm{ft}+\alpha_{0}-(\pi f \mu \sigma)^{\frac{1}{2}} \mathrm{z}\right)}
\end{aligned}
$$

Where $\mu, \sigma, J_{x 0}, J_{y 0}$ and $\alpha_{0}$ are permeability, conductivity of the sample, $x$ - and $y$ components of the induced current, and phase lag at the surface, respectively. The first exponential term in Eq. (1) shows the decay of the induced current as a function of depth $z$ within the material, i.e., the skin depth, and the depth $\delta$ of the skin effect is expressed when it decays to $J_{x 0} / e$ as:

$$
\delta=z=\sqrt{\frac{1}{\pi f \mu \sigma}}
$$


The second exponential term in Eq. (1) shows the phase angle delay of the induced eddy current concerning $z$. Moreover, it is also obvious that when the frequency $f$ is increased, $\delta$ decreases while the phase angle delay increases. The induced eddy current then can be determined by measuring the secondary magnetic field produced by the induced eddy current based of Maxwell's equation of $\operatorname{rot} \boldsymbol{H}=\boldsymbol{J}$. Since the magnetic field measurement is carried out outside the material where the eddy current is governed by the skin depth effect, this reflects that the ECT is a non-volumetric current measurement. Moreover, by manipulating the frequency of the excitation magnetic field, the penetration depth of the magnetic field can be controlled, hence different depth level of the eddy current can be investigated. To shorten the measurement time, simultaneous measurement of multiple frequency components can be used to obtain richer information of eddy current distribution at different depth, and this method has been shown in the PEC and multi-frequency techniques [15]. Compared to the conventional single frequency excitation of the ECT method, a pulse of the magnetic field which contains harmonics of the frequency components is applied to the conductive sample. This will induce a response of a pulse magnetic field generated from the eddy current where it contains the magnitude and delay information and commonly can be analysed in time and frequency domains [24].

However, it should be noted that the magnetic response also contains the effect of magnetisation properties of the material where commonly a reference signal is required to subtract this effect and isolate only the eddy current information in the PEC technique. For these reasons, the ECT technique is commonly applied for coating thickness measurement, conductivity, magnetic permeability, plate thickness measurement, surface and near-surface crack evaluation [9, 23, 25-28]. On the other hand, the PEC technique has been applied for subsurface evaluation of thick samples such as tubular structures, thickness measurements and corrosion evaluations [15, 26, 29]. It is worth to mention that utilisation of highly sensitive magnetic sensors from the DC region such as SQUIDs has enabled the low-frequency ECT technique to be used in the characterisation of subsurface and deep defects $[20,23,27,30]$.

The MFL system works by detecting the magnetic flux leakage introduced by a flaw. Commonly, to reduce the influence of magnetisation fluctuations which is problematic in the case of ECT technique, a strong magnetic field is applied by using an electromagnetic yoke to a ferromagnetic sample to saturate its magnetisation. Anomalies such as cracks in the path of the magnetic flux will produce a leakage, and this leakage can be detected by magnetic sensors. The MFL technique has shown a better detection performance not limited to surface cracks [31]. However, the use of the electromagnet yoke and high current to saturate the sample magnetisation results to a size increase of the MFL system.

On the other hand, the ECT system offers a compact system for the surface crack detection in a small and nonplanar shape of the sample. The effect of the magnetisation fluctuation can be minimised by using a planar differential detection unit such that offset fluctuations can be reduced by subtracting the detection output with a reference detection unit. Moreover, it has also been shown that the reduction the detection area under the ECT probe results to the increase of signal ratio between crack induced eddy current and the magnetisation signal from a sample [9]. The use of small magnetic sensors and excitation coils can improve the localisation performance of crack detection. In this work, based on the above considerations, we report on the development of a small eddy current probe for detection of cracks in welded parts. Moreover, since cracks can develop as low as sub-millimetre in size (micro-crack) at the surface and as a form of internal cracks, 
which may not observe by visual inspection, it is important to achieve a sensitive detection with a sub-millimetre resolution.

Furthermore, since cracks can develop from the level of microstructure, the capability to have early detection of them is important so that an early assessment can be made before they progress to a severe condition and causing accidents. For this reason, a first-order planar differential ECT probe fabricated from high sensitivity AMR sensors (HMC1001, Honeywell) is developed with a home-made instrumentation amplifier. As a preliminary step for the detection of cracks in welded parts, artificial slits on a carbon steel plate is used as a representation of crack defect for the detection purpose. The width of the artificial slits was set not larger than $1 \mathrm{~mm}$. Then, the performance of the developed ECT probe is evaluated by measuring magnetic response resulted from the artificial slits.

\section{METHODS AND MATERIALS}

\section{ECT Probe}

The schematic circuit of the developed ECT probe is shown in Figure 1. The AMR sensor is mainly composed of a resistive Wheatstone bridge of 4 AMR elements and a set/reset strap, which is wound around the AMR elements. The sensitive axis of the AMR sensor is positioned to detect the normal component of the magnetic field concerning the sample, i.e., the $B_{z}$-component. The set/reset strap is used to recover the sensitivity of the AMR sensor by re-aligning the magnetisation direction of the AMR elements towards their anisotropy axes. For this reason, re-magnetisation of the AMR elements by flowing high pulse currents through the set/reset strap are periodically required to preserve the sensitivity of the sensor. When a magnetic field is applied to the direction of the AMR sensitive axis, its resistance will change and resulting in the unbalanced voltage between the mid-points of the bridge branches. The voltage difference between these two points can be measured accurately by an instrumentation amplifier (INA) where the loading effect is reduced. Ensuring the low noise characteristic of the INA is considered important so that a high sensitivity detection unit can be achieved.

For this reason, a home-made INA is fabricated from ultra-low noise operation amplifiers of AD797 in the conventional topology of 3 operational amplifiers. A gain of 99 is chosen to achieve sufficient amplification output between $-5 \mathrm{~V}$ and $5 \mathrm{~V}$ and to preserve a wide dynamic range of the INA frequency response. A set/reset circuit is developed to apply a high-current pulse into the set/reset strap and can be physically controlled by a mechanical switch. The INAs are powered by a voltage rail-splitter of TLE2426 and the AMR sensors are biased at $5 \mathrm{~V}$ using a linear voltage regulator. The outputs of the INAs are connected to a lock-in amplifier for a phase sensitive detection in a differential mode. The excitation coil is fabricated by a $0.1-\mathrm{mm}$ diameter $\mathrm{Cu}$ wire and forms a 60-turn square coil. The excitation coil has a dimension of $11 \mathrm{~mm} \times 11 \mathrm{~mm}$ and its current is fed from the reference signal of the lock-in amplifier. The two AMR sensors are separated by a 4-mm baseline and inserted inside the excitation coil. The position of the ECT probe is determined by an $X Y$ stage and a sample can be scanned with a maximum dimension of $45 \mathrm{~cm} \times 60 \mathrm{~cm}$. 


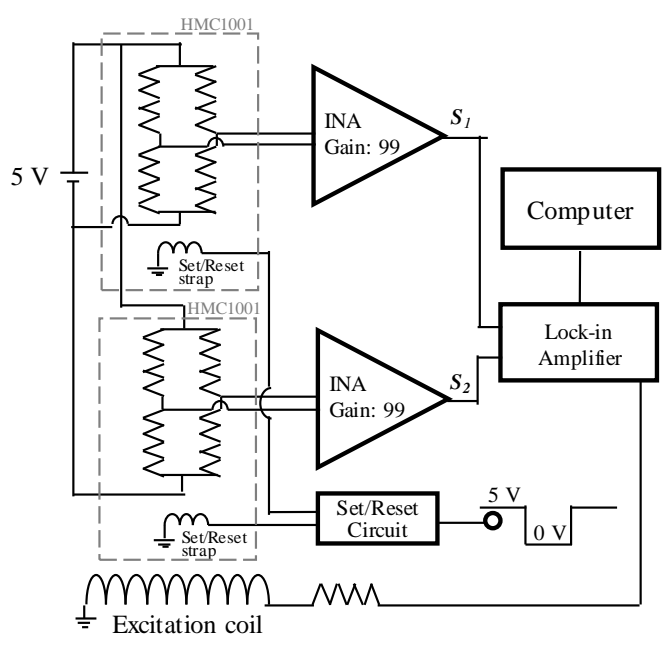

(a)

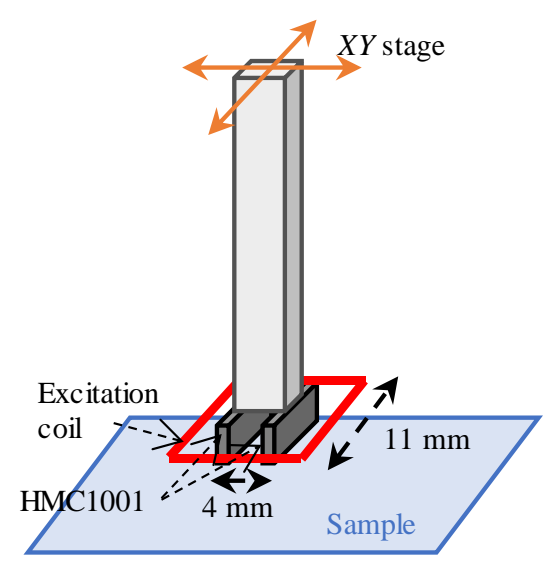

(b)

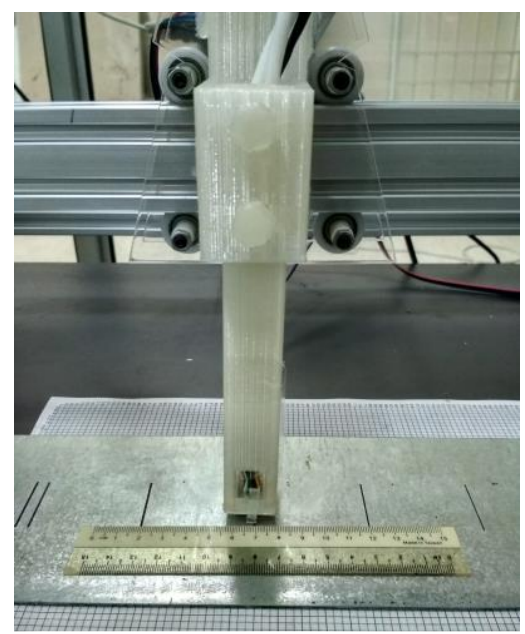

(c)

Figure 1. (a) A schematic diagram of the developed ECT probe's circuit. (b) The spatial arrangement of the AMR sensors and the excitation coil. (c) Photograph of the fabricated ECT probe.

When an AC magnetic field is applied to a ferromagnetic sample, a magnetic response $\boldsymbol{S}_{1}$ is produced and consists of a large magnetization signal $\boldsymbol{S}_{m a g, l}$ due to its magnetisation curve $M-H$ characteristic $[11,32]$ and a small eddy current induced signal $\boldsymbol{S}_{e d d y, 1}$. This can be illustrated in Figure 2. When a defect is present in the sample, a phase delay in $\boldsymbol{S}_{e d d y, l}$ is introduced where the induced eddy current takes a longer path to enclose its loop. By taking $S_{1}$ as the reference signal for the phase detection of the differential signal $\boldsymbol{S}_{1}-\boldsymbol{S}_{2}$, the phase delay of the small eddy current can be detected since the large fluctuation of $\boldsymbol{S}_{\text {mag, },}$ is minimised using the difference between two sensors. However, it should be noted that the baseline and the size of the excitation coil have a great effect to $\boldsymbol{S}_{m a g, l}$ since the distribution of magnetic property may exist between a finite distance on

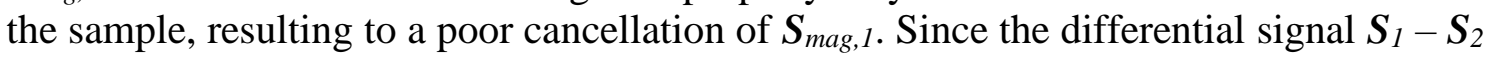
is measured at a baseline of $\Delta x=4 \mathrm{~mm}$, the differential signal of the $B_{z}$-component is equivalent to a gradient of $\Delta B_{z} / \Delta x \approx \partial B_{z} / \partial x$. 


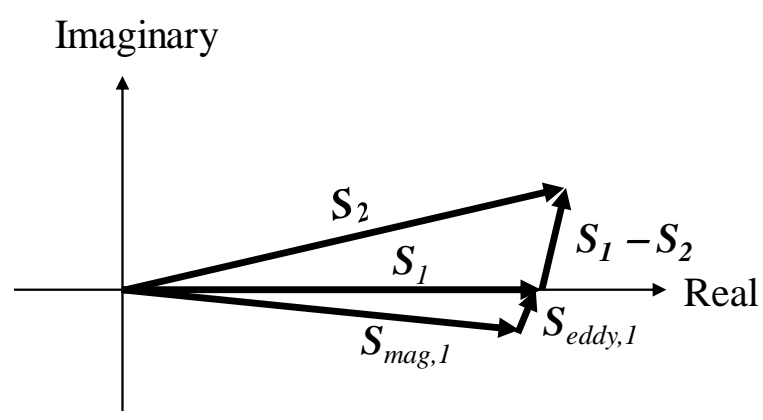

Figure 2. The differential vector of the measured magnetic signals.

\section{Carbon Steel Plate with Laser-Engraved Slits}

To test the performance of the developed ECT probe, artificial slits were laser-engraved on a 3-mm carbon steel plate. To realise the detection of sub-millimetre cracks which are hardly visible with the naked eyes [33], the width of the slits was set to be not larger than $1 \mathrm{~mm}$. The slits have depth and length of $0.3 \mathrm{~mm}$ and $30 \mathrm{~mm}$ with different widths from $0.2 \mathrm{~mm}$ to $1.0 \mathrm{~mm}$. The slits are shown in Figure 3, where they are separated by an interval of $50 \mathrm{~mm}$. It was noted that the carbon steel plate showed a non-negligible magnetic remanence, which may affect the measurement of the eddy current component.

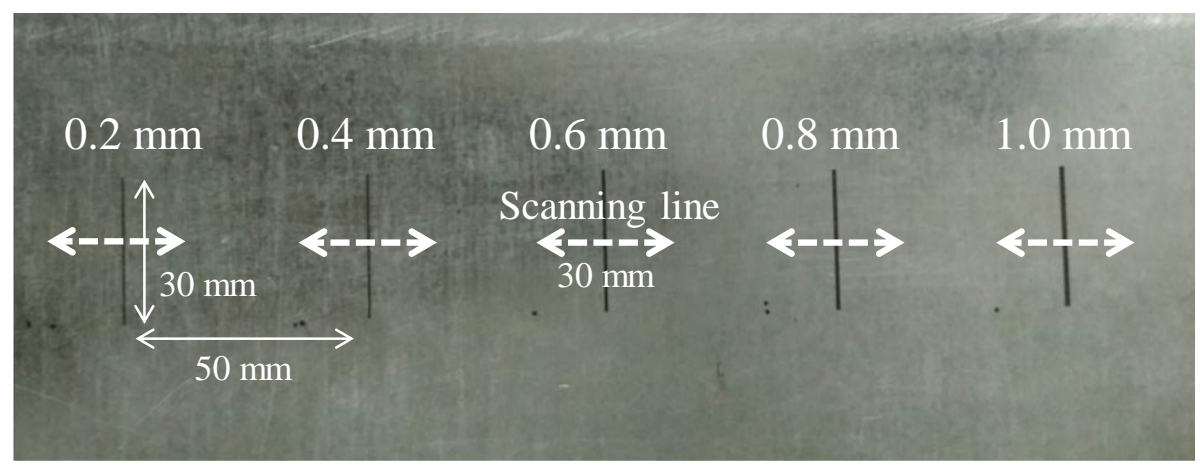

Figure 3. Artificial slits (defect) of width from $0.2 \mathrm{~mm}$ to $1 \mathrm{~mm}$ on a carbon steel plate. The white horizontal dashed line shows the line scanning direction of the ECT probe.

\section{RESULTS AND DISCUSSION}

\section{Line Scanning of Slits using ECT Probe}

Using the prepared carbon steel plate, we first measured the magnetic response from each slit by means of line scanning measurements. Figure 4 shows the real and imaginary components of the differential magnetic response when the ECT probe was moved horizontally across the slits, following the white dashed line shown in Figure 3. The ECT probe was displaced in the range of $30 \mathrm{~mm}$ with a $1-\mathrm{mm}$ interval, and the positions of the slits were set to be located at $15 \mathrm{~mm}$. The excitation magnetic field was set to be $200 \mathrm{~Hz}$ and generated by a 4-mA $\mathrm{Ap}_{\mathrm{pp}}$ current. The real component (Figure 4 (a)) showed a slight change of magnetic response around the slit position of $15 \mathrm{~mm}$.

Moreover, the imaginary component (Figure 4 (b)) revealed an apparent change of the magnetic response at the slit position compared to the real component. However, it should be noted that the intensity of the real component was higher compared to the 
imaginary component and this could be thought to result from the strong magnetisation signal of the carbon steel plate. Although the differential signal method was applied in the detection, the magnetic response of both components showed a non-constant drift characteristic. This drift characteristic might result from the distribution of the magnetic properties across the surface of the carbon steel metal and the slight variation of the liftoff between the surface and the magnetic sensors. The magnetic response change of the real component at the slit position could be interpreted as the magnetic flux leakage component, i.e., the real component is in-phase with the excitation field. The imaginary component represented the out-of-phase magnetic response, and the fluctuations of this imaginary component at the slit position could be considered as the delay and intensity change of the eddy current [11]. As shown in Figure 4 (b), the existence of the slit had resulted in the delay of the eddy current, which could be detected from the imaginary components of the measured differential magnetic responses.

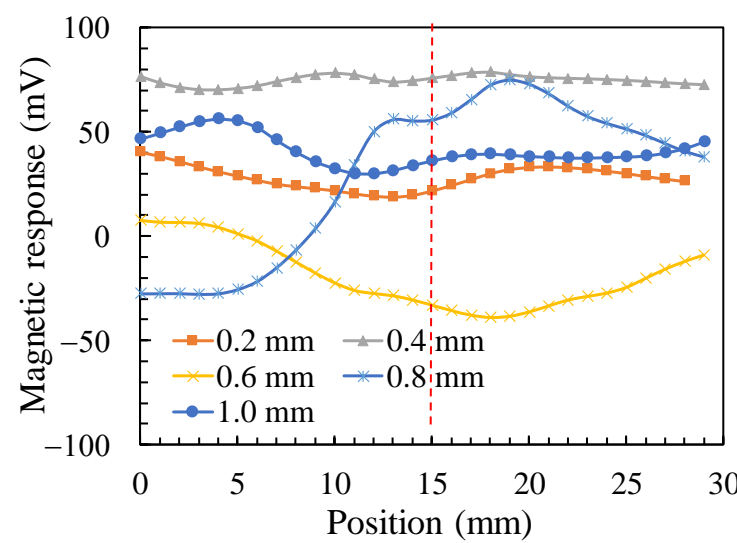

(a)

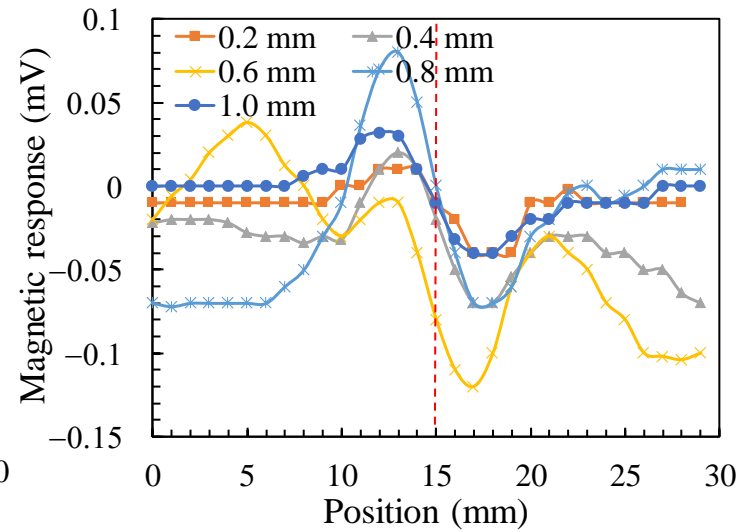

(b)

Figure 4. (a) Real and (b) imaginary components of the magnetic gradient response $\partial B_{z} / \partial x$ concerning the position of the slits during the line scanning at $200 \mathrm{~Hz}$. The scanning direction was at the centre and perpendicular to the direction of the slits. The position of the slits was set to be located at $15 \mathrm{~mm}$ (the red vertical lines).

In Figure 5, the line scanning measurement was performed at the excitation field of $10 \mathrm{kHz}$ where a higher intensity of the eddy current generation was expected at the surface of the carbon steel plate. The magnitude and characteristic of the real component was almost similar to the case of the $200-\mathrm{Hz}$ excitation field. Although a slight change in the real component was observed at the slit position, the imaginary component showed a clearer intensity change at the slit position. Compared to the case of the $200-\mathrm{Hz}$ excitation field, the imaginary component of the $0.2-\mathrm{mm}$ slit showed a stronger intensity change with less noise. Since the excitation frequency was increased by 50 times, it could be expected that the skin depth will reduce to around seven times. This might increase the eddy current density near the surface by approximately 7 times, which was in reasonable agreement with the increase of the magnetic response. The distance between the peak and trough around the slit position revealed the distance of the 4-mm baseline between the magnetic sensors, which was smaller compared to the width of the slits. Moreover, the correlation between the signal intensity change and the width of the slits was not clearly observed. This could be thought due to the larger baseline of the magnetic sensors and the dimension of the excitation coil compared to the width of the slits. 


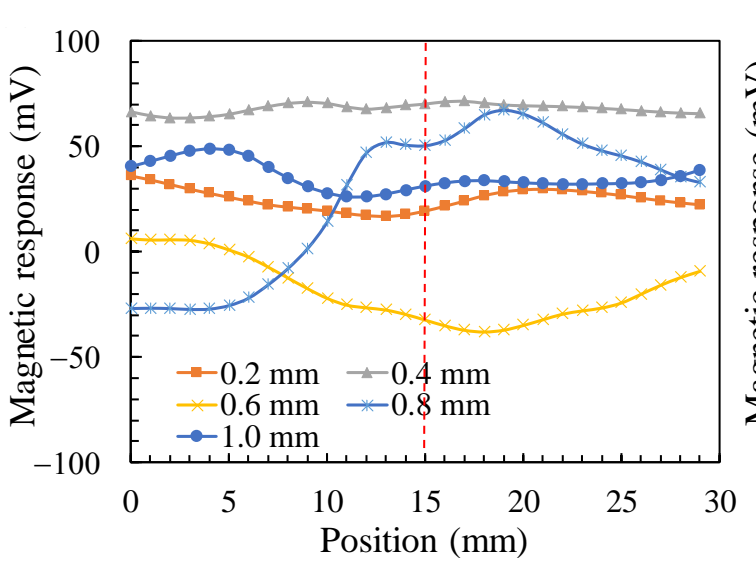

(a)

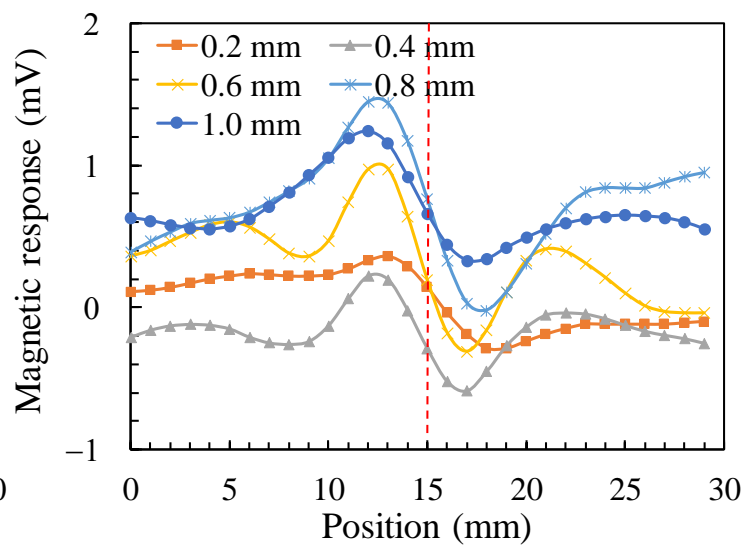

(b)

Figure 5. (a) The real and (b) imaginary components of the magnetic gradient response $\partial B_{z} / \partial x$ concerning the position of the slits during the line scanning at $10 \mathrm{kHz}$. The scanning direction was at the centre and perpendicular to the direction of the slits. The position of the slits was set to be located at $15 \mathrm{~mm}$, indicated by the red vertical lines.

\section{Magnetic Response Distribution of 0.2-mm Slit}

A measurement of the gradient magnetic response distribution across the surface of the $0.2 \mathrm{~mm}$ slit was performed at the 200-Hz excitation field. Figure 6 shows the spatial distributions of the real and imaginary components in the region of $30 \mathrm{~mm} \times 50 \mathrm{~mm}$. The magnetic gradient response was measured in the interval of $1 \mathrm{~mm}$, and the slit was positioned at $x=15 \mathrm{~mm}$ and in the $y$-direction. The intensity change due to the presence of the slit was not observed in the real component distribution. The resulted real component distribution was thought due to the distribution of magnetic properties across the plate. Although the imaginary component showed a slightly noisy response in the line scanning measurement (Figure 4 (b)), the imaginary component distribution revealed a clear intensity change at the location of the slit. This intensity change reflected the delay and density change of the eddy current caused by the slit. From Maxwell's equation rot $\boldsymbol{H}=\boldsymbol{J}$ and the Cohen-Hosaka transformation [34], the measured $\partial B_{z} / \partial x$ was proportional to the dipole current component of $-J_{y}$, where the current density of $J_{y}$ increased around the slit. This showed that the existence of the slit had directed the eddy current to be parallel with the slit.

Moreover, the length in the $y$-direction of the region where the intensity change occurred was almost similar to the length of the slit. This proves that the imaginary component distribution can be used to estimate the dimension of the defect. This result proved that the developed ECT probe was able to identify the defect based on the current dipole vector, even though in the case of the smallest slit. The capability of the ECT technique to resolve sub-millimetre defects is also shown in reference [9] where a gradient Tunnelling Magnetoresistance sensor was developed and able to detect a crack width as low as $0.3 \mathrm{~mm}$. Improvements in the design of the excitation coil can also be expected to improve the detection of sub-millimetre defects. 


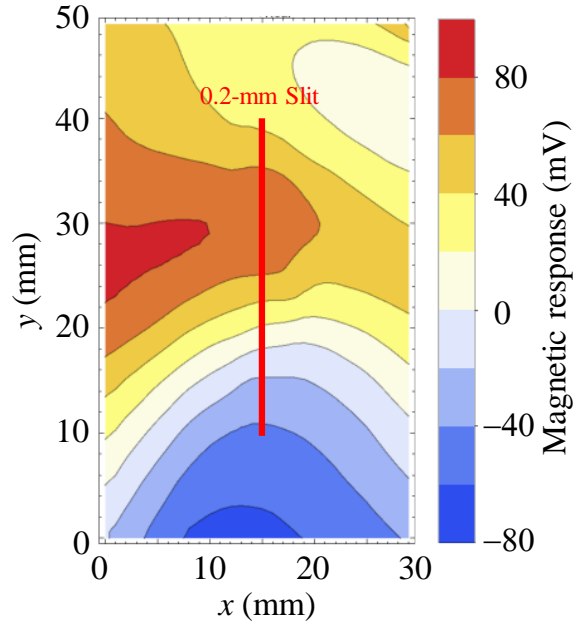

(a)

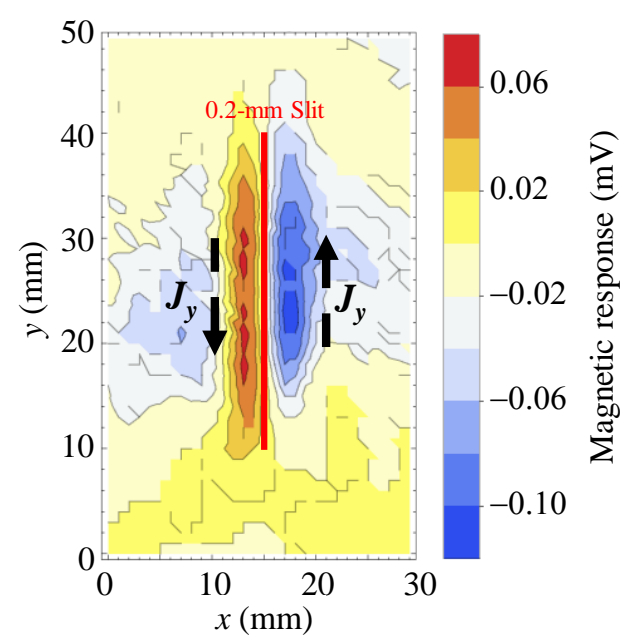

(b)

Figure 6. (a) The distributions of real and (b) imaginary component of the magnetic gradient response $\partial B_{z} / \partial x$ for the $0.2-\mathrm{mm}$ slit. The excitation magnetic field was $200 \mathrm{~Hz}$.

\section{CONCLUSION}

In this study, a small ECT probe for the surface defect evaluation in welded parts was developed. To assure a sensitive detection of the magnetic response from cracks, a planar differential probe consisted of sensitive AMR sensors and a home-made instrumentation amplifier was designed and fabricated. The performance of the developed ECT probe was evaluated by the magnetic response measurement of the sub-millimetre slits on the ferromagnetic carbon steel plate. Compared to the real component, the imaginary component of the measured differential signal could identify the presence of the slits at the excitation frequency of $200 \mathrm{~Hz}$ and $10 \mathrm{kHz}$. The distribution map of the magnetic response revealed that the signal intensity was changed around the location of the slit and could be used to estimate the dimension of the surface defect. In the future, the study is expected to be extended in the detection of cracks in welded parts and serve as a powerful tool in providing an early assessment of defects in metallic parts.

\section{ACKNOWLEDGEMENT}

The authors would like to thank the Ministry of Higher Education of Malaysia (grant no. RDU160115) and the Universiti Malaysia Pahang (grant no. RDU170377) for laboratory facilities and financial assistance.

\section{REFERENCES}

[1] Ahmad MI., Arifin A, Abdullah S. Evaluation of magnetic flux leakage signals on fatigue crack growth of mild steel. Journal of Mechanical Engineering and Sciences 2015; 9: 1727-1733.

[2] Ghoni R, Dollah M, Sulaiman A, et al. Defect Characterization Based on Eddy Current Technique: Technical Review. Advances in Mechanical Engineering; 2014. Epub ahead of print 2014.

[3] Tsukada K, Majima Y, Nakamura Y, et al. Nondestructive evaluation of defects in welded sections of rails via unsaturated AC magnetic flux leakage measurements 
with a gradiometer. $1-8$.

[4] Petcher PA, Dixon S. Weld defect detection using PPM EMAT generated shear horizontal ultrasound. NDT and E International 2015; 74: 58-65.

[5] Yusof MFM, Kamaruzaman MA, Zubair M, et al. Detection of defects on weld bead through the wavelet analysis of the acquired arc sound signal. Journal of Mechanical Engineering and Sciences 2016; 10: 2031-2042.

[6] Yaakob KI, Ishak M, Idris SRA. The effect of pulse welding parameters on weld geometry of boron steel using low power fibre laser. Journal of Mechanical Engineering and Sciences 2017; 11: 2895-2905.

[7] Shah LH, Mohamad UK, Yaakob KI, et al. Lap joint dissimilar welding of aluminum AA6061 and galvanized iron using TIG. Journal of Mechanical Engineering and Sciences (JMES) 2016; 10: 1817-1826.

[8] Hussin MH, Che Lah NA. Effects of temperature on the surface and subsurface of Al-Mg-Si welded joints. Journal of Mechanical Engineering and Sciences 2017; 11: 2743-2754.

[9] Tsukada K, Hayashi M, Nakamura Y, et al. Small Eddy Current Testing Sensor Probe Using a Tunneling Magnetoresistance Sensor to Detect Cracks in Steel Structures. IEEE Transactions on Magnetics, pp. 1-5; 2018.

[10] Dobie G, Summan R, Pierce SG, et al. A noncontact ultrasonic platform for structural inspection. IEEE Sensors Journal 2011; 11: 2458-2468.

[11] Tsukada K, Majima Y, Nakamura Y, et al. Detection of inner cracks in thick steel plates using non-saturated AC magnetic flux leakage testing with a magnetic resistance gradiometer. In 2017 IEEE International Magnetics Conference (INTERMAG), pp. 1-2; 2017.

[12] Hamia R, Cordier C, Dolabdjian C. Eddy-current non-destructive testing system for the determination of crack orientation. NDT \& E International 2014; 61: 24 28.

[13] Horan P, Underhill PR, Krause TW. Pulsed eddy current detection of cracks in F/A-18 inner wing spar without wing skin removal using Modified Principal Component Analysis. NDT \& E International 2013; 55: 21-27.

[14] Nafiah F, Sophian A. Pulsed eddy current imaging of inclined surface cracks. Indonesian Journal of Electrical Engineering and Informatics 2017; 5: 309-316.

[15] Sophian A, Tian G, Fan M. Pulsed Eddy Current Non-destructive Testing and Evaluation: A Review. Chinese Journal of Mechanical Engineering (English Edition) 2017; 30: 500-514.

[16] Kiwa T, Hayashi T, Kawasaki Y, et al. Magnetic thickness gauge using a Fourier transformed eddy current technique. NDT and E International 2009; 42: 606-609.

[17] Tsukada K, Yoshioka M, Kiwa T, et al. A magnetic flux leakage method using a magnetoresistive sensor for nondestructive evaluation of spot welds. NDT and E International 2011; 44: 101-105.

[18] Azaman KN, Sophian A, Nafiah F. Effects of Coil Diameter in Thickness Measurement Using Pulsed Eddy Current Non-destructive Testing. IOP Conference Series: Materials Science and Engineering; 260. Epub ahead of print 2017.

[19] Tsukada K, Yasugi T, Majima Y, et al. Absolute-magnetic-field measurement using nanogranular in-gap magnetic sensor with second-harmonic and liquidnitrogen-temperature operation. AIP Advances 2017; 7: 056670.

[20] Tsukada K, Kiwa T, Kawata T, et al. Low-Frequency Eddy Current Imaging Using MR Sensor Detecting Tangential Magnetic Field Components for Nondestructive 
Evaluation. IEEE Transactions on Magnetics 2006; 42: 3315-3317.

[21] He D. AMR Sensor and its Application on Nondestructive Evaluation. In: Magnetic Sensors - Development Trends and Applications. InTech, 2017. Epub ahead of print November 2017.

[22] Hatsukade Y, Shinyama Y, Yoshida K, et al. SQUID NDE on braided carbon fiber reinforced polymers with middle-end fibers under step-by-step tensile loading. IEEE Transactions on Applied Superconductivity 2013; 23: 0-4.

[23] Hatsukade Y, Tanaka S. Mobile NDE System Utilizing Robust HTS-SQUID Magnetometer for Use in Unshielded Environments. IEEE Transactions on Applied Superconductivity; 26. Epub ahead of print 2016.

[24] Kiwa T, Kawata T, Yamada H, et al. Fourier-transformed eddy current technique to visualize cross-sections of conductive materials. NDT and E International 2007; 40: 363-367.

[25] Coil HTS, Sasayama T, Ishida T, et al. Thickness Measurement of an Iron Plate Using Low-Frequency Eddy Current Testing. 2016; 26: 1-5.

[26] García-Martín J, Gómez-Gil J, Vázquez-Sánchez E. Non-destructive techniques based on eddy current testing. Sensors 2011; 11: 2525-2565.

[27] He DF, Tachiki M, Itozaki H. Highly sensitive anisotropic magnetoresistance magnetometer for Eddy-current nondestructive evaluation. Review of Scientific Instruments 2009; 80: 1-3.

[28] Chen X, Lei Y. Electrical conductivity measurement of ferromagnetic metallic materials using pulsed eddy current method. NDT \& E International 2015; 75: 3338.

[29] Xu X, Liu M, Zhang Z, et al. A Novel High Sensitivity Sensor for Remote Field Eddy Current Non-Destructive Testing Based on Orthogonal Magnetic Field. Sensors 2014; 14: 24098-24115.

[30] Kiwa T, Tahara H, Miyake E, Yamada H, Tsukada K. Non-Contact Thickness Gauge for Conductive Materials Using HTS SQUID System. IEEE Transactions on Applied Superconductivity 2009; 19: 801-803.

[31] Tsukada K, Yoshioka M, Kawasaki Y, et al. Detection of back-side pit on a ferrous plate by magnetic flux leakage method with analyzing magnetic field vector. NDT and E International 2010; 43: 323-328.

[32] Saari MM, Sakai K, Kiwa T, et al. Characterization of the magnetic moment distribution in low-concentration solutions of iron oxide nanoparticles by a high$\mathrm{T}$ c superconducting quantum interference device magnetometer. Journal of Applied Physics 2015; 117: 17B321.

[33] Verma J, Taiwade RV. Effect of welding processes and conditions on the microstructure, mechanical properties and corrosion resistance of duplex stainless steel weldments-A review. Journal of Manufacturing Processes 2017; 25: 134152.

[34] Cohen D, Hosaka H. Part II: magnetic field produced by a current dipole. Journal of Electrocardiology 1976; 9: 409-417. 